\title{
Over 30 years of research on crushed salt as a barrier material: fundamental findings and open questions
}

\author{
Kornelia Zemke, Kristoff Svensson, Ben Laurich, and Johanna Lippmann-Pipke \\ Federal Institute for Geosciences and Natural Resources (BGR), Hannover, Germany \\ Correspondence: Kornelia Zemke (kornelia.zemke@bgr.de) \\ Published: 10 November 2021
}

\begin{abstract}
Repositories for high-level radioactive waste in geological formations require knowledge on thermal, mechanical and fluid transport properties of the whole repository system, including the engineered barriers and backfill materials. For about 30 years, crushed salt has been considered the most suitable geotechnical barrier material to backfill cavities and encapsulate radioactive waste in rock salt repository sites (e.g., Czaikowski et al., 2020). Over time, when the surrounding cavity walls converge by the creep of salt, it can become strongly compacted and safely encapsulates radioactive waste from any fluid flow. Hence, crushed salt has been characterized in detail for its physical material properties and its response to environmental controls (stress, temperature and moisture). This characterisation provides a basis for long-term numerical simulations (e.g., Liu et al., 2018), which verify so-called safety cases in radioactive waste disposal.

Displacement-controlled oedometric compaction tests mimic the long-term in situ behaviour of crushed salt. The tests show that it can be compacted to a state comprising physical rock properties similar to natural rock salt. In general, compaction is easier with an increase in humidity and temperature (e.g., Stührenberg, 2007; Kröhn, et al., 2017). Triaxial test series address the compactions' response to differing confining pressures and help to identify generalized constitutive equations for crushed salt. Both BGR procedures, the oedometric and the triaxial compaction, are verified by the German accreditation body (DAkkS).

Figure 1 illustrates the history of oedometric tests at the BGR laboratory since 1993, which examined crushed salt from various origins and differing temperature conditions. Most tests focused on material from the Asse mine, revealing the compactions' response to the materials' humidity and to brine flow. Moreover, systematic test series with synthetic grain size distributions and bentonite additives provided a basis for barrier material design. More recent tests on bedded salt formations (e.g., Teutschenthal and Sondershausen mines) allow the differentiation from characteristics from domal salt deposits (e.g. Gorleben).

The current research continues the history of oedometric and triaxial tests, but has a new focus on late compaction stages with marginal remaining porosities $(<5 \%)$. The approach of systematic material characterization under best-controlled conditions essentially benefits from the international research collaboration in the KOMPASS project (Czaikowski et al., 2020). The aim of its current phase two is to synthetically generate, identify and quantify dominant grain-scale deformation processes in response to changes in environmental controls. Subsequently, these laboratory results will be embedded in numerical models on the long-term in situ rheology of crushed salt.
\end{abstract}

Kurzfassung. Endlager für hochradioaktive Abfälle in geologischen Formationen erfordern Kenntnisse über die thermischen, mechanischen und fluiden Transporteigenschaften des gesamten Endlagersystems, einschließlich der technischen Barrieren und des Verfüllmaterials. Seit etwa 30 Jahren gilt Salzgrus als das am besten geeignete geotechnische Barrierematerial für die Verfüllung von Hohlräumen und die Einkapselung radioaktiver Abfälle in Steinsalzlagerstätten (z. B. Czaikowski et al., 2020). Im Laufe der Zeit, wenn die umliegenden Hohlraumwände durch das Kriechen des Salzes konvergieren, kann es stark kompaktiert werden und die radioaktiven Abfälle sicher vor jeglichem Flüssigkeitsstrom einkapseln. Aus diesem Grund ist Salzgrus hinsichtlich seiner physikalischen Materialeigenschaften und seiner Reaktion auf Umwelteinflüsse (Druck, Temperatur und Feuchtigkeit) eingehend untersucht. Die dabei festgestellten Eigenschaften bilden die Grundlage 
für numerische Langzeitsimulationen (z. B. Liu et al., 2018), die sogenannte Sicherheitsfälle in der Entsorgung radioaktiver Abfälle verifizieren.

Weggesteuerte Kompaktionsversuche im Oedometer ahmen das langfristige In-situ-Verhalten von Salzgrus nach. Die Versuche zeigen, dass es zu einem Zustand kompaktiert werden kann, der ähnliche physikalische Gesteinseigenschaften wie natürliches Steinsalz aufweist. Im Allgemeinen ist die Kompaktion mit zunehmender Feuchtigkeit und Temperatur einfacher (z. B. Stührenberg, 2007; Kröhn et al., 2017). Triaxiale Versuchsreihen adressieren die Auswirkungen der Kompaktion auf unterschiedliche Umschließungsdrücke und helfen dabei, generalisierte konstitutive Gleichungen für Salzgrus zu ermitteln. Beide BGR-Verfahren, die oedometrische und die triaxiale Kompaktion, wurden von der Deutschen Akkreditierungsstelle (DAkkS) geprüft.

Abbildung 1 zeigt die Entwicklung der oedometrischen Versuche im BGR-Labor seit 1993, bei denen Salzgrus unterschiedlicher Herkunft und unter unterschiedlichen Temperaturbedingungen untersucht wurde. Die meisten Versuche konzentrieren sich auf den Feuchteeinfluss des Materials der Schachtanlage Asse. Außerdem lieferten systematische Versuchsreihen mit synthetischer Korngrößenverteilung und Bentonitzusätzen eine Grundlage für die Zusammenstellung von Barrierematerial. Neuere Versuche an Salzgrus aus flach lagernden Salzformationen (z. B. Grube Teutschenthal und Bergwerk Sondershausen) erlauben eine Abgrenzung zu den Eigenschaften von Salzgrus, der aus Salzstöcken gewonnen wurde (z. B. Gorleben).

Die aktuelle Forschung knüpft an die lange Geschichte der oedometrischen und triaxialen Versuche an, hat aber einen neuen Schwerpunkt auf stark kompaktiertes Salzgrus mit geringen Restporositäten $(<5 \%)$. Der Ansatz der systematischen Materialcharakterisierung unter bestkontrollierten Bedingungen profitiert wesentlich von der internationalen Forschungszusammenarbeit im KOMPASS-Projekt (Czaikowski et al., 2020). Ziel der aktuellen zweiten Phase ist die synthetische Erzeugung, Identifizierung und Quantifizierung dominanter kornskaliger Deformationsprozesse als Reaktion auf Veränderungen der Umweltbedingungen. Anschließend werden diese Laborergebnisse in numerische Modelle zur langfristigen In-situ-Rheologie von Salzgrus eingebettet.
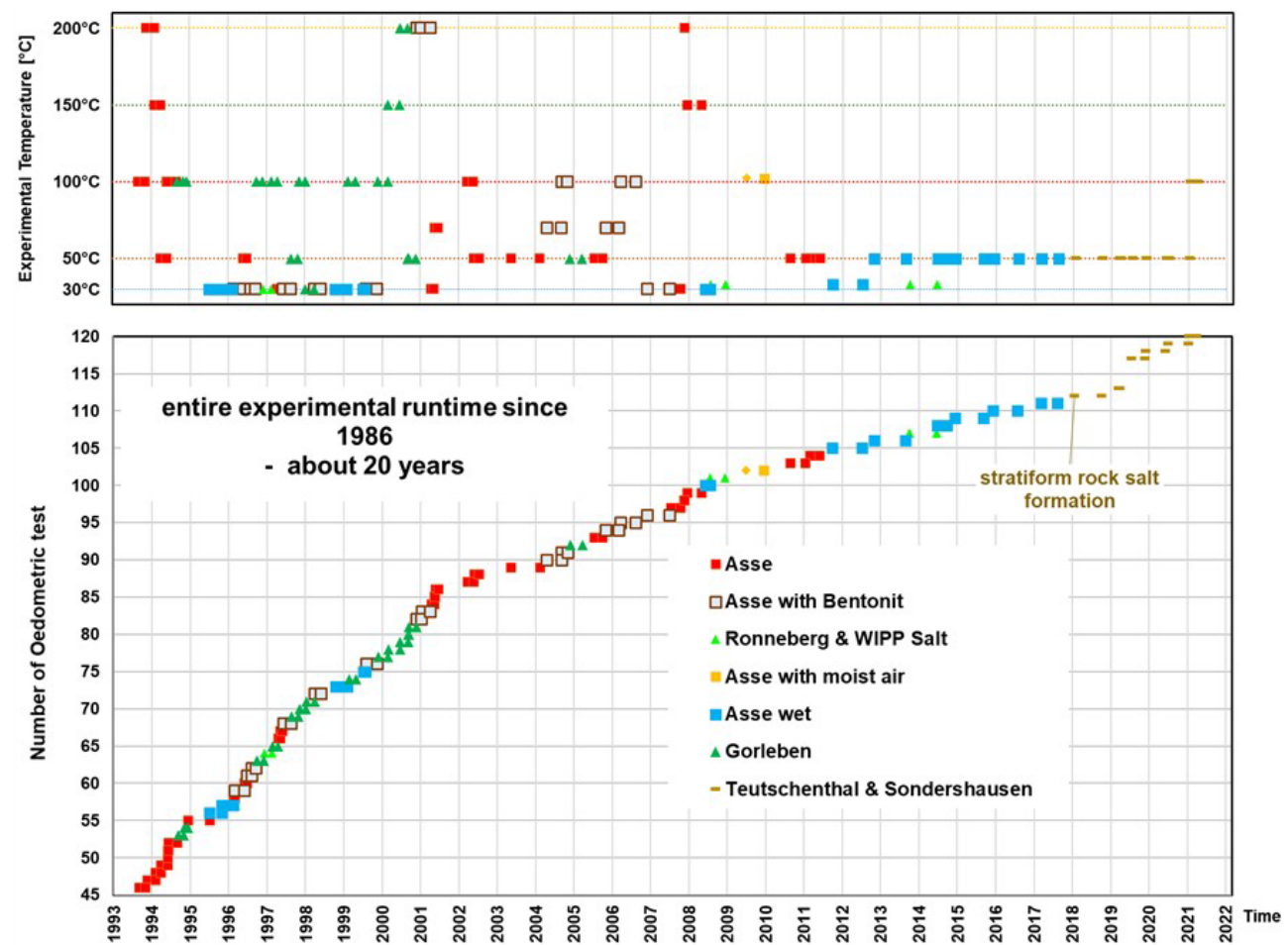

Figure 1. Tested material and respective temperature condition of oedometric tests at the BGR laboratory since 1993. 


\section{References}

Czaikowski, O., Friedenberg, L., Wieczorek, K., MüllerHoeppe, N., Lerch, C., Eickemeier, R., Laurich, B., Liu, W., Stührenberg, D., Svensson, K., Zemke, K., Lüdeling, C., Popp, T., Bean, J., Mills, M., Reedlunn, B., Düsterloh, U., Lerche, S., and Zhao, J.: KOMPASS - Compaction of crushed Salt for the safe Containment, final report, GRS-608, Braunschweig, ISBN 978-3-947685-94-3, 2020.

Kröhn, K.-P., Stührenberg, D., Jobmann, M., Heemann, U., Czaikowski, O., Wieczoerk, K., Müller, C., Zhang, C. L., Moog, H., Schirmer, S., and Friedenberg, L.: Mechanical and hydraulic behaviour of compacting crushed salt backfill at low porosities, Repoperm - Phase 2, GRS-450, ISBN 978-3-94660732-8, Gesellschaft für Anlagen- und Reaktorsicherheit (GRS) gGmbH, Braunschweig, 2017.
Liu, W., Knauth, M., Eickemeier, R., Fahland, S., Minkley, W., and Popp, T.: TM- und THM-gekoppelte Modellberechnungen zur Integritätsanalyse der geologischen Barrieren in flach lagernden Salzformationen - Ergebnisse aus dem Vorhaben KOSINA, Bundesanstalt für Geowissenschaften und Rohstoffe (BGR), Hannover und Institut für Gebirgsmechanik (IfG), Leipzig, BMWiFKZ 02E11395/02E11405, Hannover, 2018.

Stührenberg, D.: Long-term laboratory investigation on backfill The Mechanical Behavior of Salt - Understanding of the THMC Processes in Salt, in: Proceedings of "Saltmech6" Hannover, Germany, 2007. 